\title{
Development of a tool to schedule school timetabling through linear programming
}

\author{
Delmy Paola Pérez Escober, Estudiante Universitario1 \\ ıUniversidad Tecnológica Centroamericana (UNITEC), Facultad de Ingeniería, Departamento de Ingeniería Industrial y de \\ Sistemas, Tegucigalpa, Honduras, 11101, delmy_perez98@unitec.edu \\ Mentor: Daniel Montenegro, M.Sc1 \\ ıUniversidad Tecnológica Centroamericana (UNITEC), Facultad de Ingeniería, Departamento de Ingeniería Industrial y de \\ Sistemas, Tegucigalpa, Honduras, 11101, daniel.guerrero@unitec.edu.hn
}

\begin{abstract}
School timetabling is a complex activity that involves investing long hours of work, because of the different variables and constraints that are involved, and it must be done for each new school year. This research aimed to improve the time invested in timetabling at HS school through linear programming. Where four binary linear programming models were created, one for each educational stage obtaining a feasible solution in OpenSolver for each one, executing 109,920 constraints in total. Afterwards, a tool was developed in Excel to generate the timetabling automatically, which can be used by any user without having prior knowledge of linear programming. By making use of the tool, the timetable is done in 1.37 hours, reducing the time invested by $89.46 \%$ compared to the current situation.

Keywords-OpenSolver, mathematical model, preschool, elementary, high school.
\end{abstract}

Digital Object Identifier (DOI):

http://dx.doi.org/10.18687/LACCEI2020.1.1.104

ISBN: 978-958-52071-4-1 ISSN: 2414-6390

$18^{\text {th }}$ LACCEI International Multi-Conference for Engineering, Education, and Technology: "Engineering, Integration, and Alliances for a Sustainable Development" "Hemispheric Cooperation for Competitiveness and Prosperity on a Knowledge-Based Economy", July 27-31, 2020, Virtual Edition. 


\title{
Desarrollo de una herramienta para programar los horarios en un centro educativo mediante programación lineal
}

\author{
Delmy Paola Pérez Escober, Estudiante Universitario1 \\ IUniversidad Tecnológica Centroamericana (UNITEC), Facultad de Ingeniería, Departamento de Ingeniería Industrial y de \\ Sistemas, Tegucigalpa, Honduras, 11101, delmy_perez98@unitec.edu \\ Mentor: Daniel Montenegro, M.Sc1 \\ ıUniversidad Tecnológica Centroamericana (UNITEC), Facultad de Ingeniería, Departamento de Ingeniería Industrial y de \\ Sistemas, Tegucigalpa, Honduras, 11101, daniel.guerrero@unitec.edu.hn
}

Resumen- Programar los horarios de todos los grados y maestros en los centros educativos es una actividad compleja en la que se invierten largas horas de trabajo, debido a que cuenta con diferentes variables y restricciones y se debe realizar para cada año escolar. La presente investigación tuvo como objetivo mejorar los tiempos de programación de horarios en el centro educativo HS mediante programación lineal. Se crearon cuatro modelos matemáticos de programación lineal binaria, uno por nivel de escolaridad obteniendo una solución factible en OpenSolver para cada uno, cumpliendo con un total de 109,920 restricciones. Posteriormente, se desarrolló una herramienta en Excel para programar los horarios automáticamente, que puede ser utilizada por cualquier usuario sin tener un conocimiento previo sobre programación lineal. Al hacer uso de la herramienta, la programación de horarios para todos los niveles se obtiene en 1.37 horas, reduciendo el tiempo invertido en $89.46 \%$ en comparación a la situación actual.

Palabras claves-OpenSolver, modelo matemático, primaria, preescolar, secundaria.

Abstract- School timetabling is a complex activity that involves investing long hours of work, because of the different variables and constraints that are involved, and it must be done for each new school year. This research aimed to improve the time invested in timetabling at $\mathrm{HS}$ school through linear programming. Where four binary linear programming models were created, one for each educational stage obtaining a feasible solution in OpenSolver for each one, executing 109,920 constraints in total. Afterwards, a tool was developed in Excel to generate the timetabling automatically, which can be used by any user without having prior knowledge of linear programming. By making use of the tool, the timetable is done in 1.37 hours, reducing the time invested by $89.46 \%$ compared to the current situation.

Keywords-OpenSolver, mathematical model, preschool, elementary, high school.

\section{INTRODUCCIÓN}

Los centros educativos cada año escolar se ven en la obligación de realizar la programación de los horarios de clases para cada grado. Según Ángel y Duque, en la mayoría de los centros educativos la programación de los horarios se realiza de forma manual tomando en cuenta la distribución desarrollada en el año escolar anterior, procurando no hacer muchas modificaciones. Esto lo hacen sin importar el costo que conlleva una distribución no eficiente [1]. También existen costos implícitos en la elaboración de la programación, ya que la persona que la realiza invierte largas horas de trabajo.

Debido a la magnitud de la programación de horarios en centros educativos, realizarlo manualmente es una tarea muy compleja y esta propensa a tener errores [2].

En el centro educativo HS cada año escolar se realiza manualmente la programación de horarios de clases. Para dicha programación toman como referencia la programación de horarios del año escolar anterior. Este proceso es realizado por una persona del área administrativa, la cual invierte muchas horas de trabajo, que podrían ser aprovechadas para realizar otras actividades administrativas. Para obtener la programación final de los horarios de todo el centro educativo, la persona encargada en desarrollarla se demora aproximadamente 13 horas, es decir 2 días de trabajo.

Debido a la problemática anteriormente mencionada, la creación de un modelo matemático de programación lineal del problema de horario escolar que satisfaga todas las restricciones para programar los horarios de cada grado en el centro educativo HS, se podrán eliminar los errores que se producen al realizarla manualmente.

En una investigación realizada sobre la aplicación de programación lineal para la programación de horarios en un centro educativo en México, el tiempo para realizarla tomaba entre 5 a 7 horas y se logró reducir a 4 segundos [3]. Por lo tanto, desarrollar una herramienta que genere la programación de horarios automáticamente en Excel haciendo uso del modelo matemático, reduciría el tiempo para realizar la programación en el centro educativo HS.

Por lo tanto, el objetivo de esta investigación es mejorar los tiempos invertidos en la programación de horarios en el centro educativo HS mediante programación lineal. 


\section{MetodologíA}

\section{A. Modelo matemático}

"Los modelos matemáticos también son representaciones idealizadas, pero están expresados en términos de símbolos y expresiones matemáticas" [4]. Están compuestos por variables de decisión, función objetivo y restricciones. En una investigación realizada por Gunawan, Ming, \& Poh, propusieron un modelo para la programación de horarios, en el cual asignan los maestros y las clases de cada grado en un centro educativo, donde toma el valor de 1 si un maestro puede impartir una determinada materia en un bloque horario especifico y definieron todas las restricciones necesarias para cumplir con la programación de horarios de ese centro educativo [5].

Para construir los modelos matemáticos es necesario tener definido correctamente el problema. En esta investigación se recopilo toda la información necesaria para construir los modelos con las autoridades del centro educativo.

\section{B. Programación lineal}

Chase, Jacobs \& Aquilano exponen que "La programación lineal (o PL) se refiere a varias técnicas matemáticas utilizadas para asignar, en forma óptima, los recursos limitados a distintas demandas que compiten por ellos" [6]. La programación lineal está representada por un modelo matemático y el objetivo es la planeación de ciertas actividades dentro de las organizaciones [7].

Cornejo \& Mejía establecen que "Un problema de Programación Entera es un problema de programación lineal en el cual algunas de las variables, o todas, tienen que ser números enteros no negativos" [8].

Dentro de la programación lineal está el problema de horario que consiste en definir la secuencia de clases, satisfaciendo diferentes restricciones entre los estudiantes y maestro en un determinado periodo de tiempo, que por lo general es de una semana. este se divide en tres: problema de horario escolar, de exámenes y de cursos [9].

En la presente investigación se utilizó el problema de horario escolar de programación lineal para crear cuatro modelos matemáticos para cada nivel de escolaridad, definiendo las variables de decisión, función objetivo y restricciones para encontrar la programación de horarios. Se construyeron cuatro modelos debido a que los horarios varían para cada nivel de escolaridad, por lo tanto, facilita la elaboración de estos.

\section{Solución}

Los modelos de programación lineal se pueden resolver utilizando diferentes técnicas y métodos, en una investigación desarrollaron una herramienta en una hoja de cálculo que utiliza OpenSolver para la programación de horarios. Primero construyeron el modelo en la hoja de cálculo y validaron que funcionara adecuadamente para construir una interfaz que pueda ser utilizada para subir la información y sea fácil de manejar por cualquier usuario [10].

En esta investigación para la resolución del problema se utilizó el algoritmo simplex, que se basa en encontrar la solución óptima del problema a través de la búsqueda de todas las posibles soluciones [11], asimismo, se hizo uso de OpenSolver que es un complemento de Excel que puede resolver problemas más extensos de los que el solucionador de Excel ofrece para resolver los modelos de programación lineal. Para desarrollar la herramienta para programar los horarios del centro educativo HS automáticamente, se creó una interfaz en Excel haciendo uso de Visual Basic para programar las respectivas macros.

En una investigación para resolverlo utilizaron el programa GLPK en el cual el usuario tiene que tener conocimiento sobre programación lineal para obtener la programación de horarios [12], de igual manera en otra investigación sobre la programación de horarios en una universidad, hicieron uso de Lingo 13 para resolver el problema, por lo tanto, el usuario también debe de tener conocimiento sobre programación [13]. A diferencia de la presente investigación que además de resolver el problema en OpenSolver, se desarrollo una herramienta en la que fácilmente pueden obtener la programación de horarios sin la necesidad de que el usuario tenga conocimiento sobre programación lineal o programación como tal.

La validación de los modelos matemáticos se realizó mediante datos históricos, comparando los resultados obtenidos mediante el modelo y la programación de horarios del año escolar 2019-2020 la cual fue realizada manualmente. Asimismo, se validó la herramienta desarrollada con las autoridades del centro educativo HS.

\section{RESULTADOS Y ANÁLISIS}

\section{A. Caso de estudio}

El sistema de educación básica en Honduras está dividido en tres niveles de escolaridad: preescolar, primaria y secundaria. Cada uno de estos niveles está conformado por diferentes grados. En el centro educativo HS cuentan con los tres niveles, sin embargo, secundaria está dividido en dos: middle school y high school, por lo tanto, para la presente investigación se tomaron en cuenta los cuatro niveles. En la Tabla I se describen que grados forman parte de cada nivel. 
TABLA I

GRADOS POR NIVEL DE ESCOLARIDAD EN EL CENTRO EDUCATIVO HS

\begin{tabular}{|l|l|l|}
\hline Nivel & No. & Grado \\
\hline \multirow{2}{*}{ Preescolar } & 01 & Pre- Kínder \\
\cline { 2 - 3 } & 02 & Kínder \\
\hline \multirow{4}{*}{ Primaria } & 1 & Primero \\
\cline { 2 - 3 } & 2 & Segundo \\
\cline { 2 - 3 } & 3 & Tercero \\
\cline { 2 - 3 } & 4 & Cuarto \\
\cline { 2 - 3 } & 5 & Quinto \\
\hline \multirow{3}{*}{ Middle school } & 6 & Sexto \\
\cline { 2 - 3 } & 7 & Séptimo \\
\hline \multirow{2}{*}{ High school } & 9 & Octavo \\
\cline { 2 - 3 } & 10 & Noveno \\
\hline & 11 & Décimo \\
\hline
\end{tabular}

Para cada año escolar la Secretaria de Educación de Honduras brinda a los centros educativos una serie de materias que se deben impartir obligatoriamente en cada grado, así como el número de bloques horarios a impartir a la semana para cada una de las materias. De igual manera, en el centro educativo, aparte de las materias brindadas por la secretaria establecen otras materias que se van a impartir para cada grado y definen el número de bloques horarios a la semana para cada una.

En el centro educativo HS se imparten materias de lunes a viernes y la duración de cada bloque horario, así como la jornada escolar es diferente para cada nivel. Lo ideal es que las materias no se impartan más de dos veces al día, si estas cuentan con cinco o menos bloques horarios a la semana.

Los 21 maestros que actualmente laboran en el centro educativo ya tienen establecidas las materias que pueden impartir según su conocimiento. Existen maestros que imparten en dos o más niveles. Todos los maestros están contratados para toda la jornada escolar, por lo que están disponibles para ser asignados en cualquier bloque horario. Cada uno de los maestros solo puede ser asignado a 36 bloques horarios a la semana.

\section{B. Formulación del modelo de programación lineal}

Para obtener la programación de horarios para cada grado, se utilizó un modelo de programación lineal binaria. Para esto se construyeron cuatro modelos matemáticos, uno para cada nivel de escolaridad, que funcionan secuencialmente. Algunas ecuaciones pueden ser diferentes entre los modelos, sin embargo, la estructura en general es la misma.

Se definieron cinco índices para la estructura general del modelo, definiendo para cada índice los conjuntos a utilizar en cada modelo según el nivel de escolaridad, estos son los siguientes:

$i$ : son los grados que existen según el nivel de escolaridad. Los conjuntos para cada nivel son los siguientes:

$$
\begin{array}{ll}
\text { - } & \mathrm{GP}=\{01,02\} \\
\text { - } & \mathrm{GE}=\{1,2, \ldots, 6\} \\
\text { - } & \mathrm{GM}=\{7,8,9\} \\
\text { - } & \mathrm{GH}=\{10,11\}
\end{array}
$$

$j$ : está representada por los maestros que imparten materias, los conjuntos por nivel son los siguientes:

$$
\begin{array}{ll}
\text { - } & \mathrm{PP}=\{1,2,3,6\} \\
\text { - } & \mathrm{PE}=\{2,4, \ldots, 14\} \\
\text { - } & \mathrm{PM}=\{6,7,8,12,15, \ldots, 19\} \\
\text { - } & \mathrm{PH}=\{6,7,12,15, \ldots, 21\}
\end{array}
$$

Existen maestros que imparten materias para diferentes niveles, como es el caso del maestro 6, que imparte en los cuatro niveles.

$\boldsymbol{k}$ : son las materias que pueden ser impartidas por nivel, los conjuntos de todas las materias por nivel son:

$$
\begin{array}{ll}
\text { - } & \mathrm{MP}=\{1,2, \ldots, 14\} \\
\text { - } & \mathrm{ME}=\{15,16, \ldots, 80\} \\
\text { - } & \mathrm{MM}=\{81,82, \ldots, 110\} \\
& \mathrm{MH}=\{111,112, \ldots, 135\}
\end{array}
$$

Los conjuntos para las materias que se deben impartir más de cinco bloques horarios a la semana por nivel son:

- $\mathrm{MPH}=\{2,6,9,13\}$

- $\mathrm{MEH}=\{17,18,28,29,38,39,40,50,51,61,62$, $72,73,74\}$

- $\mathrm{MMH}=\{82,92,98,102,108\}$

- $\mathrm{MHH}=\{120,129\}$

$l$ : días de clases, para todos los niveles el conjunto de días es el siguiente:

$$
\text { - } \mathrm{D}=\{1,2, \ldots, 5\}
$$

$\boldsymbol{m}$ : representa los bloques horarios al día en los que se puede impartir materias, los conjuntos por nivel son los siguientes:

$$
\begin{array}{ll}
\text { - } & \mathrm{BP}=\{1,2 \ldots, 6\} \\
\text { - } & \mathrm{BE}=\{7,8, \ldots, 15\} \\
\text { - } & \mathrm{BM}=\{16,17, \ldots, 23\} \\
\text { - } & \mathrm{BH}=\{24,25, \ldots, 32\}
\end{array}
$$

Los parámetros necesarios para poder alimentar cada modelo se definen a continuación:

- $\boldsymbol{B}_{k i}$ : Número de bloques horarios obligatorios que se deben asignar de la materia $\mathrm{k}$ en el grado $\mathrm{i}$.

- $\boldsymbol{C}_{j k}$ : Conocimiento del maestro j para impartir la materia k.

- $\boldsymbol{D}_{j l m}$ : Disponibilidad del maestro j para impartir una materia en un determinado día y bloque horario.

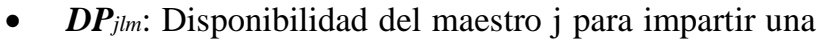
materia en un determinado día y bloque horario, tomando en cuenta la programación asignada en preescolar.

- $\boldsymbol{A c u m P}_{j:}$ : Número de bloques horarios acumulados para los maestros que imparten en primaria, si alguno de estos imparte materias en preescolar.

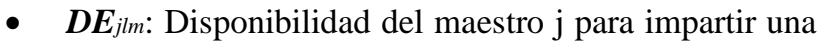
materia en un determinado día y bloque horario, tomando en cuenta la programación asignada en preescolar y primaria. 
- $\boldsymbol{A c u m E}_{j:}$ Número de bloques horarios acumulados para los maestros que imparten en middle school, si alguno de estos imparte materias en preescolar o primaria.

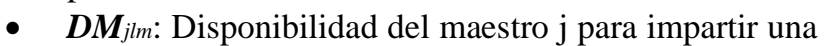
materia en un determinado día y bloque horario, tomando en cuenta la programación asignada en preescolar, primaria y middle school.

- $\operatorname{AcumM}_{j}$ : Número de bloques horarios acumulados para los maestros que imparten en high school, si alguno de estos imparte materias en prescolar, primaria o middle school.

\section{i. Modelo de preescolar}

La variable de decisión es:

$\boldsymbol{X}_{i j k l m}=$ toma el valor de 1 , si en el grado i se asigna el maestro $\mathrm{j}$ para impartir la materia $\mathrm{k}$ en el día $1 \mathrm{y}$ bloque horario $\mathrm{m}$, toma el valor de 0 en caso contrario.

La función objetivo está representada por la maximización de la asignación de los maestros y materias en todos los grados, días y bloques horarios para preescolar.

$$
\operatorname{Max} Z=\sum_{i \in G P} \sum_{j \in P P} \sum_{k \in M P} \sum_{l \in D} \sum_{m \in B P} \mathrm{Xijklm}
$$

El modelo solo está sujeto a restricciones fuertes, ya que es necesario que todas se cumplan para poder encontrar la programación de horarios para cada grado. Las restricciones son:

1. Se debe programar a lo más un maestro en cada materia, grado, día y bloque horario.

$$
\sum_{j \in P P} \mathrm{Xijklm} \leq 1 \quad \forall i \in G P, \forall k \in M P, \forall l \in D, \forall m \in B P
$$

2. Los bloques horarios programados a la semana para cada maestro no deben ser mayor a 36 bloques horarios.

$$
\sum_{i \in G P} \sum_{k \in M P} \sum_{l \in D} \sum_{m \in B P} \mathbf{X i j k l m} \leq 36 \quad \forall j \in P P
$$

3. Para los 4 maestros, solo se les puede programar una materia en la cual tenga conocimiento y disponibilidad en un bloque horario.

$\boldsymbol{X} i j l k m \leq \boldsymbol{C} j k \boldsymbol{D} j l m \quad \forall i \in G P, \forall j \in P P, \forall k \in M P, \forall l \in D, \forall m \in B P$

4. En cada bloque horario se debe programar un maestro y una materia.

$\sum_{j \in P P} \sum_{k \in M P} \mathbf{X i j k l m}=1 \quad \forall i \in G P, \forall l \in D, \forall m \in B P$
5. Solamente se puede programar una materia en un mismo bloque horario.

$$
\sum_{k \in M P} \mathbf{X i j k l m} \leq 1 \quad \forall i \in G P, \forall j \in P P, \forall l \in D, \forall m \in B P
$$

6. Las materias que son impartidas más de cinco bloques horarios a la semana deben ser programadas al menos una vez por día y máximo dos veces por día.

$1 \leq \sum_{j \in P P} \sum_{m \in B P} \mathrm{Xijklm} \leq 2 \quad \forall i \in G P, \forall k \in M P H, \forall l \in D$

7. Las materias que cuentan con cinco o menos bloques horarios a la semana deben ser programadas como máximo una vez por día.

$$
\sum_{j \in P P} \sum_{m \in B P} \mathrm{Xijklm} \leq 1 \quad \forall i \in G P, \forall k \notin M P H, \forall l \in D
$$

8. La cantidad de bloques horarios programados a la semana de una determinada materia debe ser igual a la cantidad especificada en el parámetro Bki.

$$
\sum_{j \in P P} \sum_{l \in D} \sum_{m \in B P} \mathbf{X i j k l m}_{m}=\mathbf{B} k i \quad \forall i \in G P, \forall k \in M P
$$

9. Valores permitidos para las variables.

$\boldsymbol{X} i j k l m \in(0,1) \quad \forall i \in G P, \forall j \in P P, \forall k \in M P, \forall k \in M P H, \forall k$

$$
\notin M P H, \forall l \in D, \forall m \in B P
$$

\section{ii. Modelo de primaria}

En este modelo se tomó en consideración la solución obtenida en el modelo de preescolar, ya que existen maestros que imparten materias en ambos niveles.

La variable de decisión es:

$\boldsymbol{X}_{i j k l m}=$ toma el valor de 1 , si en el grado i se asigna el maestro j para impartir la materia $\mathrm{k}$ en el día $\mathrm{l}$ y bloque horario $\mathrm{m}$, toma el valor de 0 en caso contrario.

La función objetivo está representada por la maximización de la asignación de los maestros y materias en todos los grados, días y bloques horarios para primaria.

$$
\operatorname{Max} Z=\sum_{i \in G E} \sum_{j \in P E} \sum_{k \in M E} \sum_{l \in D} \sum_{m \in B E} \mathrm{Xijklm}
$$

Al igual que en el modelo anterior, este está sujeto solamente a restricciones fuertes, ya que es necesario que todas se cumplan para poder encontrar la programación de horarios para cada grado. Las restricciones son:

1. Se debe programar a lo más un maestro en cada materia, grado, día y bloque horario. 


$$
\sum_{j \in P E} \mathrm{Xijklm} \leq 1 \quad \forall i \in G E, \forall k \in M E, \forall l \in D, \forall m \in B E
$$

2. Los bloques horarios programados a la semana para cada maestro no deben ser mayor a 36 bloques horarios.

$$
\sum_{i \in G E} \sum_{k \in M E} \sum_{l \in D} \sum_{m \in B E} \mathrm{Xijklm}+\operatorname{AcumPj} \leq 36 \quad \forall j \in P E
$$

Debido a que existen maestros que imparten materias en diferentes niveles, para los siguientes modelos las horas acumuladas serán distintas a las de este modelo.

3. Para los 12 maestros, solo se les puede programar una materia en la cual tengan conocimiento y disponibilidad en un bloque horario.

$$
\begin{aligned}
& X i j l k m \leq \boldsymbol{C} j k \boldsymbol{D P} j l m \quad \forall i \in G E, \forall j \in P E, \forall k \in M E, \forall l \in D, \forall m \\
& \in B E
\end{aligned}
$$

La disponibilidad de los maestros que imparten materias en diferentes niveles depende de la solución del primer nivel en el que imparte materias, es por esto por lo que para los siguientes modelos esta ecuación será distinta.

4. En cada bloque horario se debe programar un maestro y una materia.

$$
\sum_{j \in P E} \sum_{k \in M E} \mathbf{X i j k l m}=1 \quad \forall i \in G E, \forall l \in D, \forall m \in B E
$$

5. Solamente se puede programar una materia en un mismo bloque horario.

$$
\sum_{k \in M E} \mathrm{Xijklm} \leq 1 \quad \forall i \in G E, \forall j \in P E, \forall l \in D, \forall m \in B E
$$

6. Las materias que son impartidas más de cinco bloques horarios a la semana deben ser programadas máximo dos veces por día.

$$
\sum_{j \in P E} \sum_{m \in B E} \mathrm{Xijklm} \leq 2 \quad \forall i \in G E, \forall k \in M E H, \forall l \in D
$$

7. Las materias que tienen cinco o menos bloques horarios a la semana deben ser programadas como máximo una vez por día.

$$
\sum_{j \in P E} \sum_{m \in B E} \mathrm{Xijklm} \leq 1 \quad \forall i \in G E, \forall k \notin M E H, \forall l \in D
$$

8. La cantidad de bloques horarios programados a la semana de una determinada materia debe ser igual a la cantidad especificada en el parámetro Bki.

$$
\sum_{j \in P E} \sum_{l \in D} \sum_{m \in B E} \mathbf{X i j k l m}=\mathbf{B} k i \quad \forall i \in G E, \forall k \in M E
$$

9. Valores permitidos para las variables.

$$
\begin{aligned}
\text { Xijklm } \in(0,1) \quad & \forall i \in G E, \forall j \in P E, \forall k \in M E, \forall k \in M E H, \forall k \\
& \notin M E H, \forall l \in D, \forall m \in B E
\end{aligned}
$$

\section{iii. Modelo de middle school}

El modelo de middle school tiene la misma estructura que el modelo de primaria, a diferencia de los conjuntos. La variable de decisión y función objetivo sigue siendo la misma. Para la asignación de las variables se creó una matriz de 10,800 variables. Las ecuaciones (11), (12), (15), (16), (17), (18) y (19) del modelo de primaria se utilizaron para este modelo, solamente se modifican los conjuntos que pertenecen a cada índice, en este caso pertenecerían a los conjuntos correspondientes al modelo de middle school establecidos en la ecuación 23. Sin embargo, la (13), (14) y (20) en este modelo pasan a ser:

1. Los bloques horarios programados a la semana para cada maestro no debe ser mayor a 36 bloques horarios.

$$
\sum_{i \in G M} \sum_{k \in M M} \sum_{l \in M} \sum_{m \in B M} \text { Xijklm }+ \text { AcumE } j \leq 36 \quad \forall j \in P M
$$

2. Para los 9 maestros, solo se les puede programar una materia en la cual tenga conocimiento y disponibilidad en un bloque horario.

$\boldsymbol{X} i j l k m \leq \boldsymbol{C} j k \boldsymbol{D} \boldsymbol{E} j l m \quad \forall i \in G M, \forall j \in P M, \forall k \in M M, \forall l \in D, \forall m$ $\in B M$

3. Los valores permitidos para todas las variables en el modelo de middle school.

$\begin{aligned} X i j k l m \in(0,1) & \forall i \in G M, \forall j \in P M, \forall k \in M M, \forall k \in M M H, \forall k \\ & \notin M M H \forall l \in D, \forall m \in B M\end{aligned}$

iv. Modelo de high school

El modelo de high school tiene la misma estructura que el modelo de primaria, a diferencia de los conjuntos. La variable de decisión y función objetivo siguen siendo las mismas. Para la asignación de las variables se creó una matriz de 11,250 variables. Las ecuaciones (11), (12), (15), (16), (17), (18) y (19) del modelo de primaria se utilizaron para este modelo, solamente se modifican los conjuntos que pertenecen a cada índice, en este caso pertenecerían a los conjuntos correspondientes al modelo de high school establecidos en la ecuación 26. Sin embargo, la (13), (14) y (20) en este modelo pasan a ser: 
1. Los bloques horarios programados a la semana para cada maestro no debe ser mayor a 36 bloques horarios.

$\sum_{i \in G H} \sum_{k \in M H} \sum_{l \in M} \sum_{m \in B H}$ Xijklm + AcumM $j \leq 36 \quad \forall j \in P H$

2. Para los 10 maestros, solo se les puede programar una materia en la cual tenga conocimiento y disponibilidad en un bloque horario.

$\boldsymbol{X} i j l k m \leq \boldsymbol{C} j k \boldsymbol{D} \boldsymbol{M} j \mathrm{~lm} \quad \forall i \in G H, \forall j \in P H, \forall k \in M H, \forall l \in D, \forall m$ $\in B H$

3. Los valores permitidos para todas las variables en el modelo de high school.

Xijklm $\in(0,1) \quad \forall i \in G H, \forall j \in P H, \forall k \in M H, \forall k \in M H H, \forall k$ $\notin M H H, \forall l \in D, \forall m \in B H$

\section{Resultados}

i. Preescolar

El modelo encontró la programación factible en 0.10 minutos, cumpliendo con todas las restricciones establecidas, analizando 1,680 variables. Para representar la programación se elaboró la Tabla II donde se muestra la programación de horarios de pre- kínder, en cada bloque horario se asignó la materia y el nombre del maestro que imparte la materia.

TABLA II

PROGRAMACIÓN DE HORARIOS (PRE- KÍNDER)

\begin{tabular}{|c|c|c|c|c|c|}
\hline \\
\hline Hora & Lunes & Martes & Miércoles & Jueves & Viernes \\
\hline $7: 55-8: 30$ & $\begin{array}{c}\text { Español } \\
\text { Claudia } \\
\text { Caceres }\end{array}$ & $\begin{array}{l}\text { Vocabulary } \\
\text { Gabriela } \\
\text { Duron }\end{array}$ & $\begin{array}{l}\text { Math } \\
\text { Gabriela } \\
\text { Duron }\end{array}$ & $\begin{array}{l}\text { Reading } \\
\text { Gabriela } \\
\text { Duron }\end{array}$ & $\begin{array}{l}\text { Math } \\
\text { Gabriela } \\
\text { Duron }\end{array}$ \\
\hline $8: 30-9: 05$ & $\begin{array}{l}\text { Vocabulary } \\
\text { Gabriela } \\
\text { Duron }\end{array}$ & $\begin{array}{c}\text { Reading } \\
\text { Gabriela } \\
\text { Duron }\end{array}$ & $\begin{array}{l}\text { Español } \\
\text { Claudia } \\
\text { Caceres }\end{array}$ & $\begin{array}{c}\text { Español } \\
\text { Claudia } \\
\text { Caceres }\end{array}$ & $\begin{array}{c}\text { Vocabulary } \\
\text { Gabriela } \\
\text { Duron }\end{array}$ \\
\hline $9: 05-9: 40$ & $\begin{array}{l}\text { Reading } \\
\text { Gabriela } \\
\text { Duron }\end{array}$ & $\begin{array}{c}\text { Math } \\
\text { Gabriela } \\
\text { Duron }\end{array}$ & $\begin{array}{c}\text { P.E. } \\
\text { Dulce Andino }\end{array}$ & $\begin{array}{c}\text { Vocabulary } \\
\text { Gabriela } \\
\text { Duron }\end{array}$ & $\begin{array}{l}\text { Reading } \\
\text { Gabriela } \\
\text { Duron }\end{array}$ \\
\hline $\begin{array}{l}10: 15- \\
10: 50\end{array}$ & $\begin{array}{c}\text { Phonics } \\
\text { Gabriela } \\
\text { Duron }\end{array}$ & $\begin{array}{c}\text { Phonics } \\
\text { Gabriela } \\
\text { Duron }\end{array}$ & $\begin{array}{c}\text { Motor } \\
\text { Skills } \\
\text { Gabriela } \\
\text { Duron } \\
\end{array}$ & $\begin{array}{c}\text { Math } \\
\text { Gabriela } \\
\text { Duron }\end{array}$ & $\begin{array}{l}\text { Motor } \\
\text { Skills } \\
\text { Gabriela } \\
\text { Duron }\end{array}$ \\
\hline $\begin{array}{l}10: 50- \\
11: 25\end{array}$ & $\begin{array}{l}\text { Reading } \\
\text { Gabriela } \\
\text { Duron }\end{array}$ & $\begin{array}{c}\text { Motor } \\
\text { Skills } \\
\text { Gabriela } \\
\text { Duron }\end{array}$ & $\begin{array}{l}\text { Vocabulary } \\
\text { Gabriela } \\
\text { Duron }\end{array}$ & $\begin{array}{c}\text { Phonics } \\
\text { Gabriela } \\
\text { Duron }\end{array}$ & $\begin{array}{l}\text { Phonics } \\
\text { Gabriela } \\
\text { Duron }\end{array}$ \\
\hline $\begin{array}{l}11: 25- \\
12: 00\end{array}$ & $\begin{array}{c}\text { Motor } \\
\text { Skills } \\
\text { Gabriela } \\
\text { Duron }\end{array}$ & $\begin{array}{l}\text { Español } \\
\text { Claudia } \\
\text { Caceres }\end{array}$ & $\begin{array}{l}\text { Reading } \\
\text { Gabriela } \\
\text { Duron }\end{array}$ & $\begin{array}{c}\text { Vocabulary } \\
\text { Gabriela } \\
\text { Duron }\end{array}$ & $\begin{array}{l}\text { Español } \\
\text { Claudia } \\
\text { Caceres }\end{array}$ \\
\hline
\end{tabular}

\section{ii. Primaria}

El modelo matemático de primaria encontró la solución factible en 24.65 minutos, cumpliendo con las restricciones y tomando en consideración la programación obtenida en el modelo de preescolar. En este modelo se analizaron 35,640 variables. En la Tabla III se muestra la programación de horarios para primer grado.
TABLA III

PROGRAMACIÓN DE HORARIOS (PRIMERO)

\begin{tabular}{|c|c|c|c|c|c|}
\hline Hora & Lunes & Martes & Miércoles & Jueves & Viernes \\
\hline $7: 00-7: 40$ & $\begin{array}{l}\text { Science } \\
\text { Eloisa Funez }\end{array}$ & $\begin{array}{l}\text { Spelling } \\
\text { Eloisa Funez }\end{array}$ & $\begin{array}{c}\text { Español } \\
\text { Claudia } \\
\text { Caceres }\end{array}$ & $\begin{array}{l}\text { Grammar } \\
\text { Eloisa Funez }\end{array}$ & $\begin{array}{l}\text { Spelling } \\
\text { Eloisa Funez }\end{array}$ \\
\hline $7: 40-8: 20$ & $\begin{array}{l}\text { Music } \\
\text { Moises } \\
\text { Duron }\end{array}$ & $\begin{array}{l}\text { Grammar } \\
\text { Eloisa Funez }\end{array}$ & $\begin{array}{l}\text { Phonics } \\
\text { Eloisa Funez }\end{array}$ & $\begin{array}{c}\text { Science } \\
\text { Eloisa Funez }\end{array}$ & $\begin{array}{c}\text { Math } \\
\text { Eloisa Funez }\end{array}$ \\
\hline $8: 50-9: 30$ & $\begin{array}{c}\text { Math } \\
\text { Eloisa Funez }\end{array}$ & $\begin{array}{l}\text { Science } \\
\text { Eloisa Funez }\end{array}$ & $\begin{array}{l}\text { Reading } \\
\text { Eloisa Funez }\end{array}$ & $\begin{array}{c}\text { Estudios } \\
\text { Sociales } \\
\text { Dinora } \\
\text { Hernandez }\end{array}$ & $\begin{array}{l}\text { Reading } \\
\text { Eloisa Funez }\end{array}$ \\
\hline $9: 30-10: 10$ & $\begin{array}{l}\text { Español } \\
\text { Claudia } \\
\text { Caceres }\end{array}$ & $\begin{array}{l}\text { Reading } \\
\text { Eloisa Funez }\end{array}$ & $\begin{array}{c}\text { Math } \\
\text { Eloisa Funez }\end{array}$ & $\begin{array}{l}\text { Computer } \\
\text { Rina Flores }\end{array}$ & $\begin{array}{c}\text { Español } \\
\text { Claudia } \\
\text { Caceres }\end{array}$ \\
\hline $10: 10-10: 50$ & $\begin{array}{c}\text { Español } \\
\text { Claudia } \\
\text { Caceres }\end{array}$ & $\begin{array}{c}\text { Math } \\
\text { Eloisa Funez }\end{array}$ & $\begin{array}{l}\text { Español } \\
\text { Claudia } \\
\text { Caceres }\end{array}$ & $\begin{array}{c}\text { Español } \\
\text { Moises } \\
\text { Duron }\end{array}$ & $\begin{array}{l}\text { Grammar } \\
\text { Eloisa Funez }\end{array}$ \\
\hline $10: 50-11: 30$ & $\begin{array}{c}\text { Math } \\
\text { Eloisa Funez }\end{array}$ & $\begin{array}{l}\text { Phonics } \\
\text { Eloisa Funez }\end{array}$ & $\begin{array}{l}\text { Grammar } \\
\text { Eloisa Funez }\end{array}$ & $\begin{array}{c}\text { P.E. } \\
\text { Dulce Andino }\end{array}$ & $\begin{array}{l}\text { Music } \\
\text { Moises } \\
\text { Duron }\end{array}$ \\
\hline $12: 00-12: 40$ & $\begin{array}{c}\text { Estudios } \\
\text { Sociales } \\
\text { Dinora } \\
\text { Hernandez }\end{array}$ & $\begin{array}{l}\text { P.E. } \\
\text { Dulce } \\
\text { Andino }\end{array}$ & $\begin{array}{l}\text { Spelling } \\
\text { Eloisa Funez }\end{array}$ & $\begin{array}{l}\text { Español } \\
\text { Claudia } \\
\text { Caceres }\end{array}$ & $\begin{array}{c}\text { Estuidos } \\
\text { Sociales } \\
\text { Dinora } \\
\text { Hernandez }\end{array}$ \\
\hline $12: 40-1: 20$ & $\begin{array}{l}\text { Grammar } \\
\text { Eloisa Funez }\end{array}$ & $\begin{array}{c}\text { Estudios } \\
\text { Sociales } \\
\text { Dinora } \\
\text { Hernandez }\end{array}$ & $\begin{array}{l}\text { Science } \\
\text { Eloisa Funez }\end{array}$ & $\begin{array}{c}\text { Math } \\
\text { Eloisa Funez }\end{array}$ & $\begin{array}{c}\text { Math } \\
\text { Eloisa Funez }\end{array}$ \\
\hline $1: 20-2: 00$ & $\begin{array}{l}\text { Reading } \\
\text { Eloisa Funez }\end{array}$ & $\begin{array}{l}\text { Español } \\
\text { Claudia } \\
\text { Caceres }\end{array}$ & $\begin{array}{c}\text { Estudios } \\
\text { Sociales } \\
\text { Dinora } \\
\text { Hernandez }\end{array}$ & $\begin{array}{l}\text { Reading } \\
\text { Eloisa Funez }\end{array}$ & $\begin{array}{l}\text { Science } \\
\text { Eloisa Funez }\end{array}$ \\
\hline
\end{tabular}

iii. Middle school

El modelo matemático de middle school encontró la solución factible en 1.77 minutos, analizando 10,800 variables. Cumplió con las restricciones definidas y considerando la programación obtenida tanto en preescolar como en primaria. En la Tabla IV se muestra la programación de horarios de séptimo grado.

TABLA IV

\begin{tabular}{|c|c|c|c|c|c|}
\hline \multicolumn{6}{|c|}{ PROGRAMACIÓN DE HORARIOS (SÉPTIMO) } \\
\hline Hora & Lunes & Martes & Miércoles & Jueves & Viernes \\
\hline $\begin{array}{l}7: 00- \\
7: 45\end{array}$ & $\begin{array}{c}\text { Math } \\
\text { Bessy } \\
\text { Rodriguez }\end{array}$ & $\begin{array}{c}\text { English } \\
\text { Nancy Bueso }\end{array}$ & $\begin{array}{c}\text { Estudios } \\
\text { Sociales } \\
\text { Cinthia } \\
\text { Gonzalez }\end{array}$ & $\begin{array}{c}\text { Science } \\
\text { Manuel } \\
\text { Gutierrez }\end{array}$ & $\begin{array}{l}\text { Computer } \\
\text { Rina Flores }\end{array}$ \\
\hline $\begin{array}{l}7: 45- \\
8: 30\end{array}$ & $\begin{array}{c}\text { P.E. } \\
\text { Dulce Andino }\end{array}$ & $\begin{array}{c}\text { Music } \\
\text { Moises Duron }\end{array}$ & $\begin{array}{c}\text { Science } \\
\text { Manuel } \\
\text { Gutierrez }\end{array}$ & $\begin{array}{c}\text { Math } \\
\text { Bessy } \\
\text { Rodriguez }\end{array}$ & $\begin{array}{c}\text { Music } \\
\text { Moises Duron }\end{array}$ \\
\hline $\begin{array}{l}8: 30- \\
9: 15\end{array}$ & $\begin{array}{l}\text { Computer } \\
\text { Rina Flores }\end{array}$ & $\begin{array}{c}\text { Math } \\
\text { Bessy } \\
\text { Rodriguez }\end{array}$ & $\begin{array}{c}\text { English } \\
\text { Nancy Bueso }\end{array}$ & $\begin{array}{c}\text { English } \\
\text { Nancy Bueso }\end{array}$ & $\begin{array}{c}\text { P.E. } \\
\text { Dulce Andino }\end{array}$ \\
\hline $\begin{array}{l}9: 45- \\
10: 30\end{array}$ & $\begin{array}{c}\text { Social } \\
\text { Studies } \\
\text { Nancy Galeas }\end{array}$ & $\begin{array}{c}\text { Social } \\
\text { Studies } \\
\text { Nancy Galeas }\end{array}$ & $\begin{array}{c}\text { English } \\
\text { Nancy Bueso }\end{array}$ & $\begin{array}{c}\text { English } \\
\text { Nancy Bueso }\end{array}$ & $\begin{array}{c}\text { Español } \\
\text { Estephany } \\
\text { Gonzalez }\end{array}$ \\
\hline $\begin{array}{l}10: 30- \\
11: 15\end{array}$ & $\begin{array}{c}\text { Español } \\
\text { Estephany } \\
\text { Gonzalez }\end{array}$ & $\begin{array}{c}\text { Science } \\
\text { Manuel } \\
\text { Gutierrez }\end{array}$ & $\begin{array}{c}\text { Music } \\
\text { Moises Duron }\end{array}$ & $\begin{array}{l}\text { Computer } \\
\text { Rina Flores }\end{array}$ & $\begin{array}{c}\text { Science } \\
\text { Manuel } \\
\text { Gutierrez }\end{array}$ \\
\hline $\begin{array}{l}11: 15- \\
12: 00\end{array}$ & $\begin{array}{c}\text { Estudios } \\
\text { Sociales } \\
\text { Cinthia } \\
\text { Gonzalez }\end{array}$ & $\begin{array}{c}\text { Español } \\
\text { Estephany } \\
\text { Gonzalez }\end{array}$ & $\begin{array}{c}\text { Math } \\
\text { Bessy } \\
\text { Rodriguez }\end{array}$ & $\begin{array}{c}\text { Music } \\
\text { Moises Duron }\end{array}$ & $\begin{array}{c}\text { English } \\
\text { Nancy Bueso }\end{array}$ \\
\hline $\begin{array}{l}12: 30- \\
1: 15\end{array}$ & $\begin{array}{c}\text { Science } \\
\text { Manuel } \\
\text { Gutierrez }\end{array}$ & $\begin{array}{l}\text { Computer } \\
\text { Rina Flores }\end{array}$ & $\begin{array}{l}\text { Computer } \\
\text { Rina Flores }\end{array}$ & $\begin{array}{c}\text { Estudios } \\
\text { Sociales } \\
\text { Cinthia } \\
\text { Gonzalez }\end{array}$ & $\begin{array}{c}\text { Math } \\
\text { Bessy } \\
\text { Rodriguez }\end{array}$ \\
\hline $\begin{array}{l}1: 15- \\
2: 00\end{array}$ & $\begin{array}{c}\text { Cívica } \\
\text { Cinthia } \\
\text { Gonzalez }\end{array}$ & $\begin{array}{c}\text { Cívica } \\
\text { Cinthia } \\
\text { Gonzalez }\end{array}$ & $\begin{array}{c}\text { Español } \\
\text { Estephany } \\
\text { Gonzalez }\end{array}$ & $\begin{array}{c}\text { Español } \\
\text { Estephany } \\
\text { Gonzalez }\end{array}$ & $\begin{array}{c}\text { English } \\
\text { Nancy Bueso }\end{array}$ \\
\hline
\end{tabular}

iv. High school

El modelo matemático de high school encontró la solución factible en 1.83 minutos, analizando 11,250 variables. Cumplió con todas las restricciones definidas y considerando la programación obtenida en los tres niveles previos. En la Tabla $\mathrm{V}$ se muestra la programación de horarios de décimo grado. 
TABLA V

PROGRAMACIÓN DE HORARIOS (DÉCIMO)

\begin{tabular}{|c|c|c|c|c|c|}
\hline Hora & Lunes & Martes & Miércoles & Jueves & Viernes \\
\hline $\begin{array}{l}7: 00- \\
7: 45\end{array}$ & $\begin{array}{c}\text { Español } \\
\text { Estephany } \\
\text { Gonzalez }\end{array}$ & $\begin{array}{l}\text { Frances } \\
\text { Melany } \\
\text { Molina }\end{array}$ & $\begin{array}{l}\text { English } \\
\text { Karla Nuñez }\end{array}$ & $\begin{array}{c}\text { Math } \\
\text { Bessy } \\
\text { Rodriguez }\end{array}$ & $\begin{array}{c}\text { Español } \\
\text { Estephany } \\
\text { Gonzalez }\end{array}$ \\
\hline $\begin{array}{l}7: 45- \\
8: 30\end{array}$ & $\begin{array}{c}\text { Math } \\
\text { Bessy } \\
\text { Rodriguez }\end{array}$ & $\begin{array}{c}\text { Physics } \\
\text { Manuel } \\
\text { Gutierrez }\end{array}$ & $\begin{array}{c}\text { Philosophy } \\
\text { Cinthia } \\
\text { Gonzalez }\end{array}$ & $\begin{array}{c}\text { Sociology } \\
\text { Cinthia } \\
\text { Gonzalez }\end{array}$ & $\begin{array}{l}\text { Frances } \\
\text { Melany } \\
\text { Molina }\end{array}$ \\
\hline $\begin{array}{l}8: 30- \\
9: 15\end{array}$ & $\begin{array}{c}\text { Philosophy } \\
\text { Cinthia } \\
\text { Gonzalez }\end{array}$ & $\begin{array}{c}\text { Chemistry } \\
\text { Manuel } \\
\text { Gutierrez }\end{array}$ & $\begin{array}{c}\text { Español } \\
\text { Estephany } \\
\text { Gonzalez }\end{array}$ & $\begin{array}{c}\text { English } \\
\text { Karla Nuñez }\end{array}$ & $\begin{array}{c}\text { Sociology } \\
\text { Cinthia } \\
\text { Gonzalez }\end{array}$ \\
\hline $\begin{array}{l}9: 45- \\
10: 30\end{array}$ & $\begin{array}{l}\text { Chemistry } \\
\text { Manuel } \\
\text { Gutierrez }\end{array}$ & $\begin{array}{c}\text { Biology } \\
\text { Manuel } \\
\text { Gutierrez }\end{array}$ & $\begin{array}{l}\text { Sociology } \\
\text { Cinthia } \\
\text { Gonzalez }\end{array}$ & $\begin{array}{l}\text { Español } \\
\text { Estephany } \\
\text { Gonzalez }\end{array}$ & $\begin{array}{c}\text { Psychology } \\
\text { Cinthia } \\
\text { Gonzalez }\end{array}$ \\
\hline $\begin{array}{l}10: 30- \\
11: 15\end{array}$ & $\begin{array}{c}\text { Biology } \\
\text { Manuel } \\
\text { Gutierrez }\end{array}$ & $\begin{array}{l}\text { Computer } \\
\text { Rina Flores }\end{array}$ & $\begin{array}{c}\text { Biology } \\
\text { Manuel } \\
\text { Gutierrez }\end{array}$ & $\begin{array}{c}\text { English } \\
\text { Karla Nuñez }\end{array}$ & $\begin{array}{c}\text { Math } \\
\text { Bessy } \\
\text { Rodriguez }\end{array}$ \\
\hline $\begin{array}{l}11: 15- \\
12: 00\end{array}$ & $\begin{array}{l}\text { English } \\
\text { Karla Nuñez }\end{array}$ & $\begin{array}{c}\text { Math } \\
\text { Bessy } \\
\text { Rodriguez }\end{array}$ & $\begin{array}{c}\text { Chemistry } \\
\text { Manuel } \\
\text { Gutierrez }\end{array}$ & $\begin{array}{c}\text { Philosophy } \\
\text { Cinthia } \\
\text { Gonzalez }\end{array}$ & $\begin{array}{c}\text { P.E. } \\
\text { Dulce Andino }\end{array}$ \\
\hline $\begin{array}{l}12: 30- \\
1: 15\end{array}$ & $\begin{array}{l}\text { Computer } \\
\text { Rina Flores }\end{array}$ & $\begin{array}{c}\text { Español } \\
\text { Estephany } \\
\text { Gonzalez }\end{array}$ & $\begin{array}{c}\text { Math } \\
\text { Bessy } \\
\text { Rodriguez }\end{array}$ & $\begin{array}{c}\text { Frances } \\
\text { Melany } \\
\text { Molina }\end{array}$ & $\begin{array}{c}\text { Biology } \\
\text { Manuel } \\
\text { Gutierrez }\end{array}$ \\
\hline $\begin{array}{l}1: 15- \\
2: 00\end{array}$ & $\begin{array}{c}\text { P.E. } \\
\text { Dulce Andino }\end{array}$ & $\begin{array}{l}\text { English } \\
\text { Karla Nuñez }\end{array}$ & $\begin{array}{c}\text { English } \\
\text { Karla Nuñez }\end{array}$ & $\begin{array}{l}\text { Physics } \\
\text { Manuel } \\
\text { Gutierrez }\end{array}$ & $\begin{array}{l}\text { Physics } \\
\text { Manuel } \\
\text { Gutierrez }\end{array}$ \\
\hline $\begin{array}{l}2: 00- \\
2: 45\end{array}$ & $\begin{array}{l}\text { Physics } \\
\text { Manuel } \\
\text { Gutierrez }\end{array}$ & $\begin{array}{c}\text { Psychology } \\
\text { Cinthia } \\
\text { Gonzalez }\end{array}$ & $\begin{array}{c}\text { Psychology } \\
\text { Cinthia } \\
\text { Gonzalez }\end{array}$ & $\begin{array}{l}\text { Computer } \\
\text { Rina Flores }\end{array}$ & $\begin{array}{c}\text { Chemistry } \\
\text { Manuel } \\
\text { Gutierrez }\end{array}$ \\
\hline
\end{tabular}

Como se muestra en los resultados, en la Tabla II, III, IV y V, se observa el ejemplo de la maestra Dulce Andino que imparte la materia P.E. en los cuatro niveles educativos y en ninguna ocasión existe un choque de bloques horarios.

\section{Validación}

Para validar la programación de horarios obtenida mediante programación lineal, primero se comparó la programación obtenida con la programación realizada manualmente para el año escolar 2019- 2020.

Para la comparación se tomaron en cuenta los siguientes dos criterios:

1. Las materias que cuenten con más de cinco bloques horarios obligatorios a la semana sean programadas al menos una vez por día y máximo dos veces.

2. Las materias que tengan cinco o menos bloques horarios obligatorios a la semana sean programadas como máximo una vez por día.

Al realizar la programación de horarios manualmente del año escolar 2019-2020, se puede observar que no se cumplen los dos criterios a excepción de primero, cuarto y onceavo grado, en el primer criterio no cumplieron 19 veces y en el segundo criterio a excepción de kínder, no cumplieron 67 veces. Con la programación obtenida mediante el modelo, el primer criterio no se cumple en primero, segundo, tercero, sexto, séptimo, noveno y décimo grado, violando en total 8 veces el criterio 1, sin embargo, para el segundo criterio en todos los grados se cumple que las materias estén programadas al menos una vez por día.

En relación con las materias asignadas en cada grado, tanto en la programación realizada manualmente como en la obtenida mediante el modelo matemático, ambas cumplen con el número de bloques horarios obligatorios, ya que para que el sistema del centro educativo funcione adecuadamente estas se deben cumplir obligatoriamente.

\section{E. Herramienta para programar los horarios}

La herramienta fue desarrollada para obtener la programación de horarios para cada uno de los grados de los diferentes niveles automáticamente. Los cuatro modelos matemáticos descritos anteriormente fueron construidos en hojas de cálculo en Microsoft Excel. Una vez que se construyó y se obtuvo una solución factible, se desarrolló la herramienta que puede ser utilizada por cualquier usuario sin tener un conocimiento previo sobre programación lineal.

Los supuestos considerados para crear la herramienta son con base en la programación de horarios del año escolar 2019. 2020, estos son los siguientes:

- Las materias que se imparten en cada grado ya están establecidas.

- La cantidad de maestros que imparten materias en el centro educativo son veintiuno.

- Cada maestro tiene un perfil creado con las materias que puede impartir, por lo tanto, en dado caso que algún maestro tenga que salir del centro educativo, se buscaría un maestro que tenga el perfil del que se fue.

- La cantidad de bloques horarios que se deben programar a la semana de una determinada materia puede variar para cada año escolar.

La interfaz construida se muestra en la Fig.1.

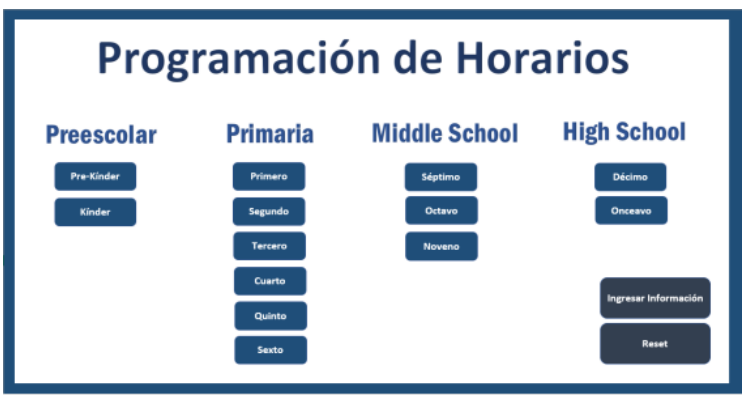

Fig. 1 Herramienta desarrollada para programar los horarios

Todos los botones tienen asignada una macro, cada botón muestra lo siguiente:

- El botón "Ingresar información" está vinculado a la hoja de cálculo para ingresar la información de preescolar, una vez que termine, el usuario dará clic en "ingresar" el cual tiene una macro vinculada para solucionar el modelo matemático y luego hará clic en "primaria" para ingresar la información correspondiente y así sucesivamente. Cuando termine de llenar todas las matrices para los cuatros niveles de escolaridad regresara a la hoja de cálculo "programación".

- Los botones con el respectivo grado tienen vinculado una macro para mostrar la programación de horarios respectiva, como se muestra para pre- kínder en la Fig.2. En cada programación existe un botón para regresar a la hoja de cálculo "programación". 


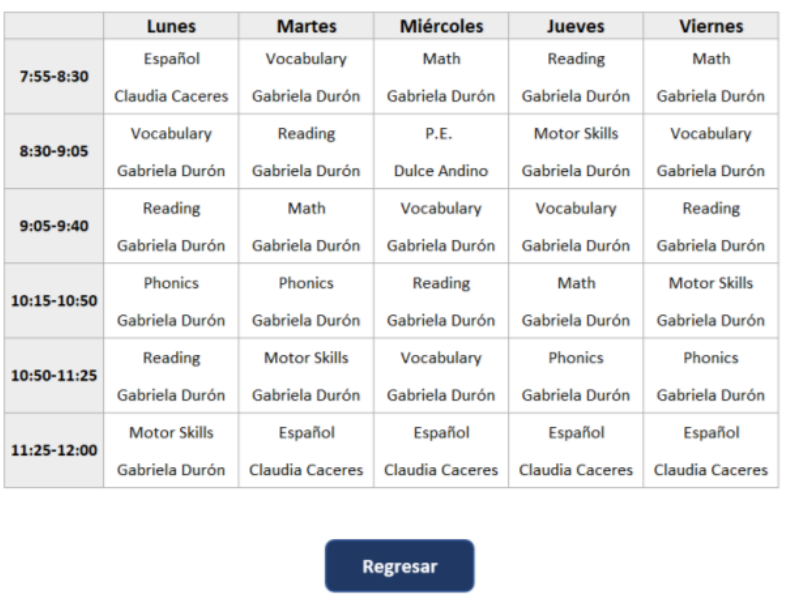

Fig. 2 Programación de horarios Pre- Kínder

Con la herramienta se obtiene la programación de horarios para todos los grados en los diferentes niveles de escolaridad en 1.37 horas. En la herramienta el tiempo de solución de los modelos aumenta debido a que se consideraron los 21 maestros para todos los niveles de escolaridad, independientemente si imparte materias en un determinado nivel o no, por lo que OpenSolver analizaba más variables en cada modelo. Sin embargo, debido a que la herramienta se construyó con las necesidades específicas del centro educativo HS, en cuanto a la flexibilidad de esta para ser utilizada en otros centros educativos se tendrá que tomar en cuenta los requerimientos que manejan al momento de programar los horarios escolares y capacitar a un usuario para que manualmente pueda actualizar ciertas restricciones según sus necesidades.

Para validar el funcionamiento de la herramienta desarrollada, se programó una reunión con la directora del centro educativo HS, donde se le presento la herramienta y como funciona. Posteriormente se realizó una entrevista con las siguientes preguntas:

1. ¿La herramienta es amigable para el usuario?

2. ¿Qué ventajas mira al utilizar la herramienta?

3. ¿Qué agregaría a la herramienta?

4. ¿Considera que la herramienta puede ser implementada en el centro educativo?

\section{CONCLUSIONES}

1. Se encontró una solución factible para la programación de horarios para cada nivel de escolaridad en el centro educativo HS, cumpliendo con 4,068 restricciones en el modelo de preescolar, 78,108 en el modelo de primaria, 13,719 en el modelo de middle school y 14,025 en el modelo de high school.

2. Al realizar la comparación entre los dos criterios, se obtuvo que, en el primer criterio, al programar los horarios mediante el modelo matemático se mejora en un 58\% con relación a la programación realizada manualmente y en un $100 \%$ en el segundo criterio.

3. Se desarrolló una herramienta amigable para que cualquier usuario sin tener un conocimiento previo sobre programación lineal pueda obtener la programación de horarios para todos los grados del centro educativo HS. Al realizar la programación con la herramienta el tiempo invertido se reduce en un $89.46 \%$.

\section{EVOLUCIÓN DE TRABAJO FUTURO}

Para darle continuidad a esta investigación, para el modelo matemático se recomienda crear un solo modelo para los cuatro niveles de escolaridad para que no exista prioridad en la disponibilidad de horarios de los maestros para impartir materias en un determinado bloque horario. Asimismo, se puede considerar las distancias que existen entre cada grado para reducir los tiempos que les toma a los maestros que imparten en diferentes niveles en llegar a cada grado.

En el caso de la herramienta, para un trabajo futuro existen diferentes oportunidades de mejora. Como habilitar la opción para que el usuario pueda ingresar cuales son las materias que se van a impartir para cada grado y cuáles son las materias que va a impartir cada maestro en un año escolar en particular. De igual manera, agregar una opción para añadir una sección a cualquier grado, en caso de que sea necesario abrir dos secciones por grado.

\section{REFERENCIAS}

[1] Ángel, J. C. M., \& Duque, P. A. M. (2016). Modelo lineal para la programación de clases en una institución educativa. Ingeniería y Ciencia; Medellín, 12(23), 47-71. http://dx.doi.org/10.17230/ingciencia.12.23.3

[2] Hernández, R. (2008). Programación de Horarios de Clases y Asignación de Salas para la Facultad de Ingeniería de la Universidad Diego Portales Mediante un Enfoque de Programación Entera. 21.

[3] Sánchez, D., \& Canseco, A. (2016). APLICACIÓN DE PROGRAMACIÓN LINEAL PARA LA ASIGNACIÓN DE HORARIOS EN UNA INSTITUCIÓN EDUCATIVA MEXICANA.

[4] Hillier, F. S., \& Lieberman, G. J. (2010). Introducción a la Investigación de Operaciones (9a edición). México: McGRAWHILL/INTERAMERICANA EDITORES, S.A. DE C.V.

[5] Gunawan, A., Ming, K., \& Lengo Poh, K. (2006). A Mathematical Programming Model For A Timetabling Problem. CSC.

[6] Chase, R. B., Jacobs, F. R., \& Aquilano, N. J. (2009). Administracion de operaciones. Producción y cadena de suministros (Duodécima edición). México, D.F: McGRAW-HILL / INTERAMERICANA EDITORES, S.A. DE C.V.

[7] Cuycaposa Rojas, J. (2016). Optimización en la programación de horarios de editores y asignación de islas de edición, para la postproducción de programas de un canal de televisión en Lima, aplicando programación lineal entera. Repositorio de Tesis - UNMSM.

[8] Cornejo, C., \& Mejía, M. (2005). MODELO DE PROGRAMACIÓN LINEAL ENTERA MIXTA PARA EL PLANEAMIENTO DE LAS IMPORTACIONES EN RÉGIMEN ADUANERO DEFINITIVO. Industrial Data, 8, 056. https://doi.org/10.15381/idata.v8i2.6188

[9] Ribeiro, G., \& Nogueira, L. A. (2006). An Integer Programming Model for the School Timetabling Problem. 6.

[10] Marmolejo, J. A., Aranzabal, I., Tellaeche, K., \& Sanchez, S. (2016). Development of a tool for university timetabling using an integrated spreadsheet. 11. 
[11] Callazo, A. (s. f.). Método simplex de PL. Recuperado 10 de junio de 2020, de https://es.scribd.com/document/144804522/Metodo-Simplex

[12] Ribic, S., \& Konjicija, S. (2010, julio 24). A two phase integer linear programming approach to solving the school timetable problem. 651-656.

[13] Sánchez-Partida, D., Martínez-Flores, J. L., \& Olivares-Benítez, E. (2014). An integer linear programming model for a university timetabling problem considering time windows and consecutive periods. 6(3), 16. 